\title{
Correction to: Comparison of a one- and two-stage mixed model analysis of Australia's National Variety Trial Southern Region wheat data
}

\author{
Beverley Gogel $\cdot$ Alison Smith • Brian Cullis
}

Published online: 13 February 2018

(C) Springer Science+Business Media B.V., part of Springer Nature 2018

\section{Correction to: Euphytica (2018) 214:44}

https://doi.org/10.1007/s10681-018-2116-4

This article has been published with an erroneous version of Eq. 15. Please find the correct Eq. 15 in this document.

Derivation of (11)

Let $\boldsymbol{T}=\left[\begin{array}{ll}\boldsymbol{T}_{1} & \boldsymbol{T}_{2}\end{array}\right]$ be an $(n \times n)$ non-singular transformation matrix such that $\boldsymbol{T}_{1}$ and $\boldsymbol{T}_{2}$, of dimension $(n \times t)$ and $(n \times(n-t))$, satisfy

$$
\begin{aligned}
& \boldsymbol{T}_{1}^{\top} \boldsymbol{X}=\boldsymbol{I}_{t} \boldsymbol{T}_{2}^{\top} \\
& \boldsymbol{X}=\mathbf{0} \Leftrightarrow \mathcal{R}\left(\boldsymbol{T}_{2}\right) \perp \mathcal{R}(\boldsymbol{X}) .
\end{aligned}
$$

Likewise, let $\boldsymbol{Q}=\left[\begin{array}{ll}\boldsymbol{Q}_{1} & \boldsymbol{Q}_{2}\end{array}\right]$ be an $((n-t) \times$ $(n-t))$ non-singular transformation matrix such that $\boldsymbol{Q}_{1}$ and $\boldsymbol{Q}_{2}$, of dimension $((n-t) \times d)$ and $((n-$ $t) \times(n-t-d))$, satisfy

The original article can be found online at https:// doi.org/10.1007/s10681-018-2116-4.

B. Gogel ( $\square)$

School of Agriculture, Food and Wine, The University of Adelaide, Waite Campus, PMB 1, Glen Osmond,

SA 5064, Australia

e-mail: beverley.gogel@adelaide.edu.au

\section{A. Smith · B. Cullis}

School of Mathematics and Applied Statistics, Faculty of Engineering and Information Sciences, University of Wollongong, Wollongong, NSW 2522, Australia

$$
\begin{aligned}
& \boldsymbol{Q}_{1}^{\top} \boldsymbol{T}_{2}^{\top} \boldsymbol{X}_{g}=\boldsymbol{I}_{d} \\
& \boldsymbol{Q}_{2}^{\top} \boldsymbol{T}_{2}^{\top} \boldsymbol{X}_{g}=\mathbf{0} \Leftrightarrow \mathcal{R}\left(\boldsymbol{Q}_{2}\right) \perp \mathcal{R}\left(\boldsymbol{T}_{2}^{\top} \boldsymbol{X}_{g}\right) .
\end{aligned}
$$

Should read:

Derivation of (11)

Let $\boldsymbol{T}=\left[\begin{array}{ll}\boldsymbol{T}_{1} & \boldsymbol{T}_{2}\end{array}\right]$ be an $(n \times n)$ non-singular transformation matrix such that $\boldsymbol{T}_{1}$ and $\boldsymbol{T}_{2}$, of dimension $(n \times t)$ and $(n \times(n-t))$, satisfy

$\boldsymbol{T}_{1}^{\top} \boldsymbol{X}=\boldsymbol{I}_{t}$

$\boldsymbol{T}_{2}^{\top} \boldsymbol{X}=\mathbf{0} \Leftrightarrow \mathcal{R}\left(\boldsymbol{T}_{2}\right) \perp \mathcal{R}(\boldsymbol{X})$.

Likewise, let $\boldsymbol{Q}=\left[\begin{array}{ll}\boldsymbol{Q}_{1} & \boldsymbol{Q}_{2}\end{array}\right]$ be an $((n-t)$ $\times(n-t))$ non-singular transformation matrix such that $\boldsymbol{Q}_{1}$ and $\boldsymbol{Q}_{2}$, of dimension $((n-t) \times d)$ and $((n-$ t) $\times(n-t-d))$, satisfy

$$
\begin{aligned}
& \boldsymbol{Q}_{1}^{\top} \boldsymbol{T}_{2}^{\top} \boldsymbol{X}_{g}=\boldsymbol{I}_{d} \\
& \boldsymbol{Q}_{2}^{\top} \boldsymbol{T}_{2}^{\top} \boldsymbol{X}_{g}=\mathbf{0} \Leftrightarrow \mathcal{R}\left(\boldsymbol{Q}_{2}\right) \perp \mathcal{R}\left(\boldsymbol{T}_{2}^{\top} \boldsymbol{X}_{g}\right) .
\end{aligned}
$$

